\title{
Evolution of Hemicellulose Molar Mass During Softwood Hydrolysis
}

\author{
Jingqian Chen ${ }^{1}$, D. M. Martinez ${ }^{1}$, Xue Feng Chang ${ }^{2}$, Rodger P. Beatson ${ }^{2}$, and \\ Heather L. Trajano ${ }^{1, *}$ \\ ${ }^{1}$ Dept. of Chemical and Biological Engineering, University of British Columbia \\ ${ }^{2}$ Chemical and Environmental Technology, British Columbia Institute of Technology \\ ${ }^{1 * C o r r e s p o n d i n g ~ A u t h o r s ~ E-m a i l: ~ h e a t h e r . t r a j a n o @ u b c . c a ~}$
}

\section{Cover sheet}

1. The number of pages: 19

2. The number of figures: 10

3. The number of tables: 5

\section{List of Figures}

S1 Logarithm of calibration standard molar mass plotted against SEC reten-

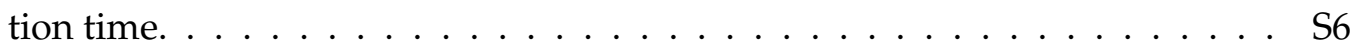

S2 SEC chromatograph of RI detector signal of hydrolysate at $180{ }^{\circ} \mathrm{C}$ for varying residence time. . . . . . . . . . . . . . . S6 
S3 Concentration calibration curve of (a) Std 1, (b) Std 3, (c) Std 5, (d) Std 7,

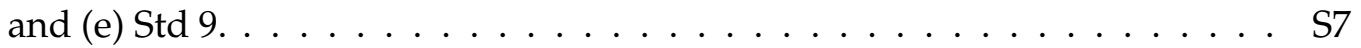

S4 A schematic illustration of pseudo-time calculation method. . . . . . . . S S8

S5 Average pseudo-time $\left(t_{p}, \mathrm{~min}\right)$ for heating and cooling as a function of temperature. Error bars are the standard deviation of $2-3$ replicates. . . . . . . . S8

S6 Mechanism of hemicellulose molar mass evolution in the range of 8 intervals including soluble oligomer with DP1-120, insoluble softwood hemicellulose, and degradation products. $P$ is a primary pathway to produce soluble hemicellulose from insoluble wood; $S$ is a secondary pathway to produce smaller soluble oligomers from a primary oligomer. . . . . . . . . S9

S7 Hydrolyzed solids (a) Acid insoluble residue (AIR) relative to initial AIR and (b) Acid soluble lignin (ASL) relative to initial ASL in $25 \mathrm{~g}$ of o.d. softwood chip fines at $160^{\circ} \mathrm{C}, 180^{\circ} \mathrm{C}$ and $200^{\circ} \mathrm{C}$ as a function of pseudo-time. Error bars are the standard deviation of $2-3$ replicates. . . . . . . . . . . . . S11

S8 Hydrolysate $\mathrm{pH}$ as a function of pseudo-time (min) and temperature. Error bars are the standard deviation of $2-3$ replicates. . . . . . . . . . . . . . . S15

S9 Hemicellulose mass recovery (summation of hydrolysate hemicellulose and prehydrolyzed solids hemicellulose) from hydrolysate and pretreated solids at $160^{\circ} \mathrm{C}$ to $200^{\circ} \mathrm{C} \mathrm{(a)} \mathrm{Galactan} \mathrm{(b)} \mathrm{Arabinan} \mathrm{(c)} \mathrm{Xylan} \mathrm{(d)} \mathrm{Glucan} \mathrm{(e)} \mathrm{Man-}$ nan. Error bars are the standard deviation of 2-3 replicates. . . . . . . . . . . S16

S10 Linear regression for activation energy determination of (a) interval 8, (b) interval 1 and 2, (c) interval 3, (d) interval 4, (e) interval 5, (f) interval 6, and (g) interval 7. . . . . . . . . . . . . . . . . . . S17 


\section{List of Tables}

S1 Carbohydrates hydrolysis by population balance model ( $k$ and $h$ are hydrolysis rate constant; $a, b$ are constant) . . . . . . . . . . . . S4

S2 Calibration standard retention time and molar mass. . . . . . . . . . . S5

S3 Molar mass and SEC retention time by DP. . . . . . . . . . . . . . . . S10

S4 Composition of softwood on o.d. biomass basis and average hemicellulose monomer molar mass. The standard deviation is calculated from 4-6 replicates of each batch. . . . . . . . . . . . . . . S12

S5 Comparison of hemicellulose/wood hydrolysis activation energies reported in literature. . . . . . . . . . . . . . . . . . . . S18 
Table S1: Carbohydrates hydrolysis by population balance model ( $k$ and $h$ are hydrolysis rate constant; $a, b$ are constant).

\begin{tabular}{|c|c|c|c|c|c|}
\hline Year & 1978 & 2008 & 2012 & 2015 & 2015 \\
\hline Author & $\begin{array}{l}\text { Basedow et al. } \\
(1978)\end{array}$ & Xu et al. (2008) & Visuri et al. (2012) & $\begin{array}{l}\text { Greenwood et al. } \\
(2015)\end{array}$ & Ahmad et al. (2015) \\
\hline Substrate & Dextran & $\begin{array}{l}\text { O-Acetyl Galactogluco- } \\
\text { mannans }\end{array}$ & Galactoglucomannan & Sugarcane bagasse & Eucalyptus kraft pulp cellulose \\
\hline $\begin{array}{l}\text { Hydrolysis condi- } \\
\text { tion }\end{array}$ & $\begin{array}{l}0.12 \mathrm{~N} \text { sulfuric } \\
\text { acid }\end{array}$ & $1 \mathrm{M} \mathrm{HCl} \mathrm{pH}$ of $1-2$ & Hot water $\mathrm{pH}$ of $3.8-4.2$ & $0.5 \%$ sulfuric acid & Alkaline solution with oxygen \\
\hline Temperature $\left({ }^{\circ} \mathrm{C}\right)$ & 80 & $90,70,50,37$, and 25 & $150,160,170$ & $110-170$ & 50 \\
\hline Model & $\begin{array}{l}\text { Pseudo- } \\
\text { homogeneous }\end{array}$ & Pseudo-homogeneous & Population balance model & $\begin{array}{l}\text { Population balance } \\
\text { model }\end{array}$ & Population balance model \\
\hline $\begin{array}{l}\text { Bond breaking } \\
\text { mode }\end{array}$ & End wise & Random & $\begin{array}{l}\text { Random/fast-slow bond } \\
\text { competition }\end{array}$ & Random & Random \\
\hline $\begin{array}{l}\text { Oligomer charac- } \\
\text { terization }\end{array}$ & $\begin{array}{l}\text { Number of } \\
\text { bonds }\end{array}$ & Number of bonds & Number of bonds & $\begin{array}{l}\text { Oligomer concen- } \\
\text { tration }\end{array}$ & Particle concentration \\
\hline Rate equation & Rate $=k(D P)^{a}$ & Rate $=k_{w 0} k_{w}(T) N_{t}(D P-1)$ & N.A. & N.A. & N.A. \\
\hline Selection function & N.A. & N.A. & $\begin{array}{l}S=h\left[H^{+}\right](D P-1) \\
h_{1} \text { is constant or } \\
h_{2}=a k_{\text {fast }}+(1-a) k_{\text {slow }}\end{array}$ & $S=k\left[H^{+}\right]$ & $\begin{array}{l}S_{1}=k_{1}(D P-1) \\
S_{2}=k_{2}(D P-1) \exp \left(-a_{2} t\right) \\
S_{3}=k_{3}(D P-1)^{b_{3}} \\
S_{4}=k_{4}(D P-1)^{b_{4}} \exp \left(-a_{4} t\right)\end{array}$ \\
\hline
\end{tabular}


Table S2: Calibration standard retention time and molar mass.

\begin{tabular}{lccc}
$\begin{array}{l}\text { Calibration } \\
\text { standard \# }\end{array}$ & $\begin{array}{c}\text { Retention } \\
\text { time (min) }\end{array}$ & Mw (Da) & LogMw \\
\hline Std1 & 42.14 & 20,600 & 4.31 \\
Std2 & 43.63 & 12,600 & 4.10 \\
Std3 & 45.73 & 6,690 & 3.83 \\
Std4 & 47.50 & 4,290 & 3.63 \\
Std5 & 51.45 & 1,400 & 3.15 \\
Std6 & 52.88 & 1,030 & 3.01 \\
Std7 & 55.01 & 633 & 2.80 \\
Std8 & 56.85 & 430 & 2.63 \\
Std9 & 60.29 & 202 & 2.31 \\
Std10 & 61.84 & 106 & 2.03 \\
\hline
\end{tabular}




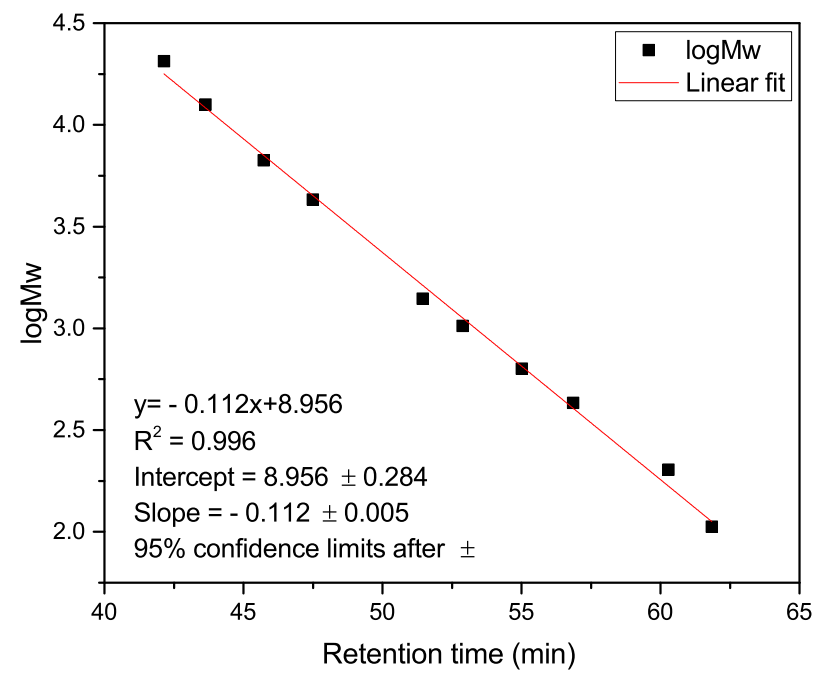

Figure S1: Logarithm of calibration standard molar mass plotted against SEC retention time.

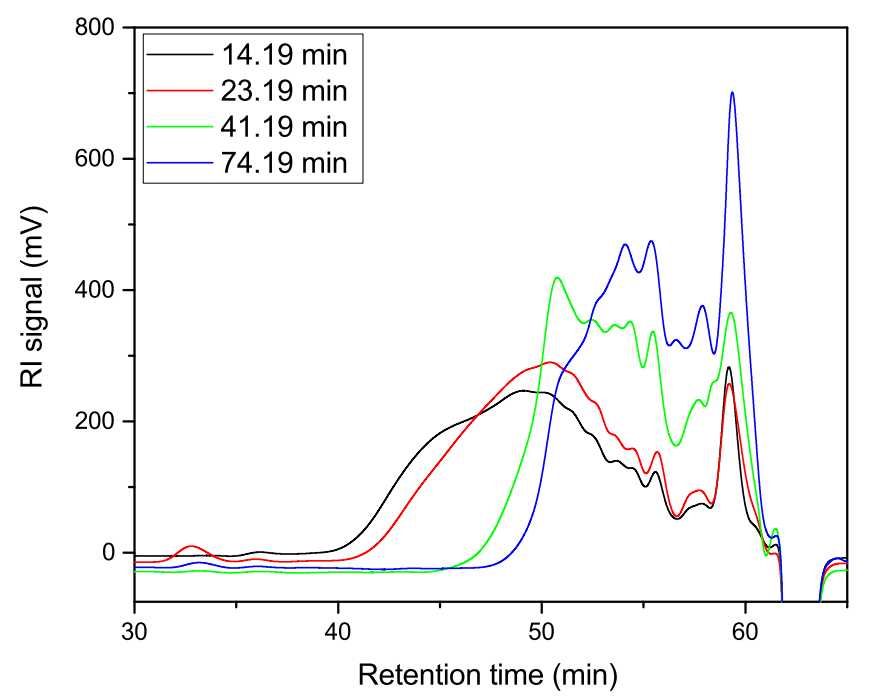

Figure S2: SEC chromatograph of RI detector signal of hydrolysate at $180{ }^{\circ} \mathrm{C}$ for varying residence time. 

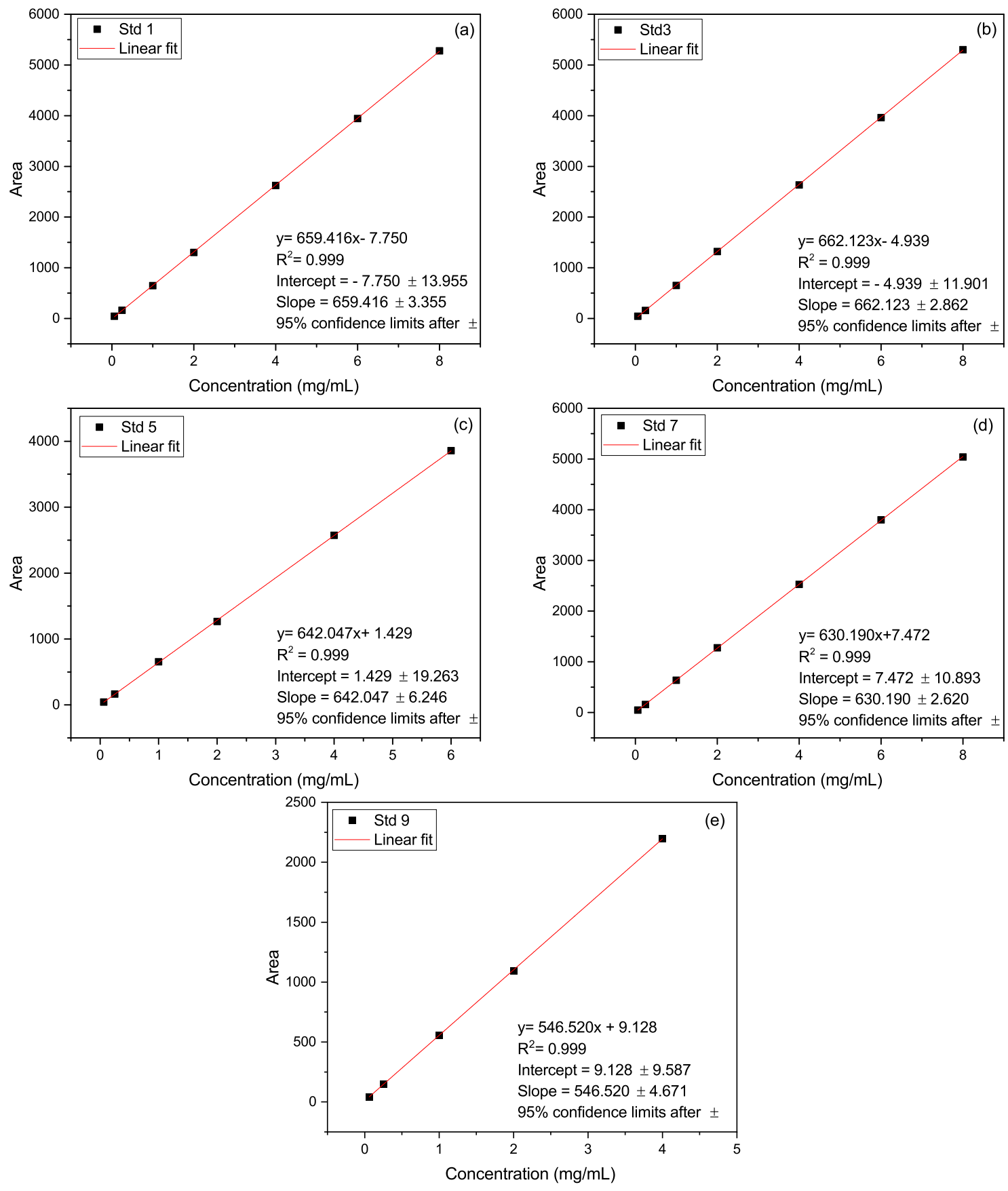

Figure S3: Concentration calibration curve of (a) Std 1, (b) Std 3, (c) Std 5, (d) Std 7, and (e) Std 9. 
The pseudo-time for heating and cooling was calculated according to Equation 1, and presented as a function of temperature in Figure S5.

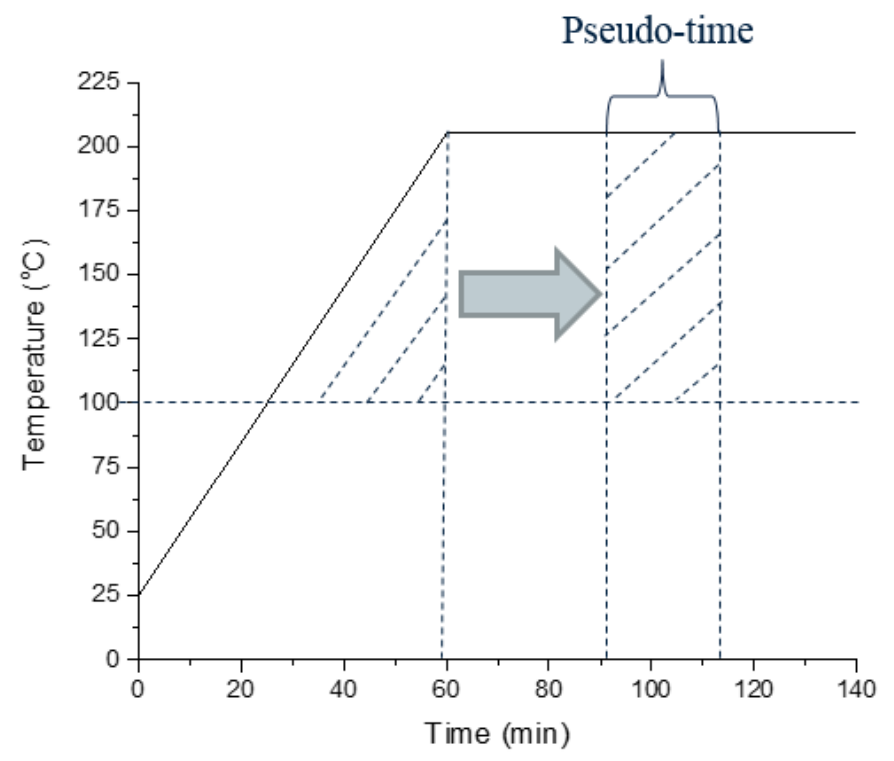

Figure S4: A schematic illustration of pseudo-time calculation method.

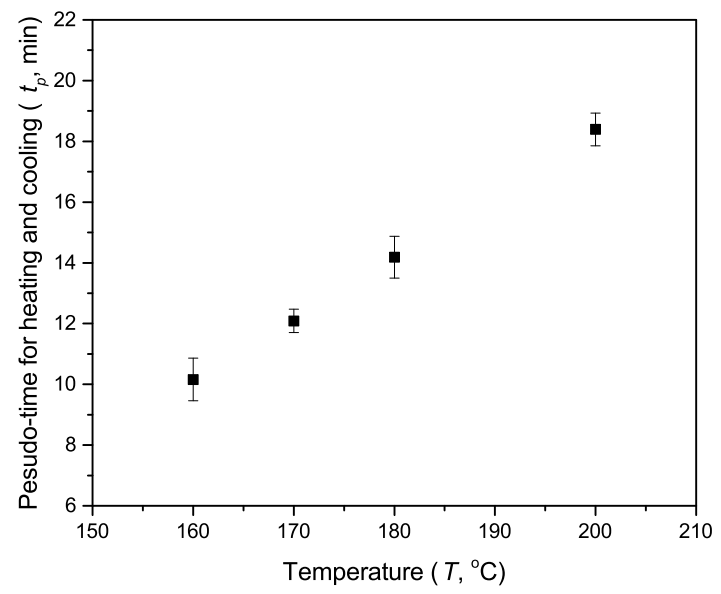

Figure S5: Average pseudo-time $\left(t_{p}, \mathrm{~min}\right)$ for heating and cooling as a function of temperature. Error bars are the standard deviation of 2-3 replicates. 


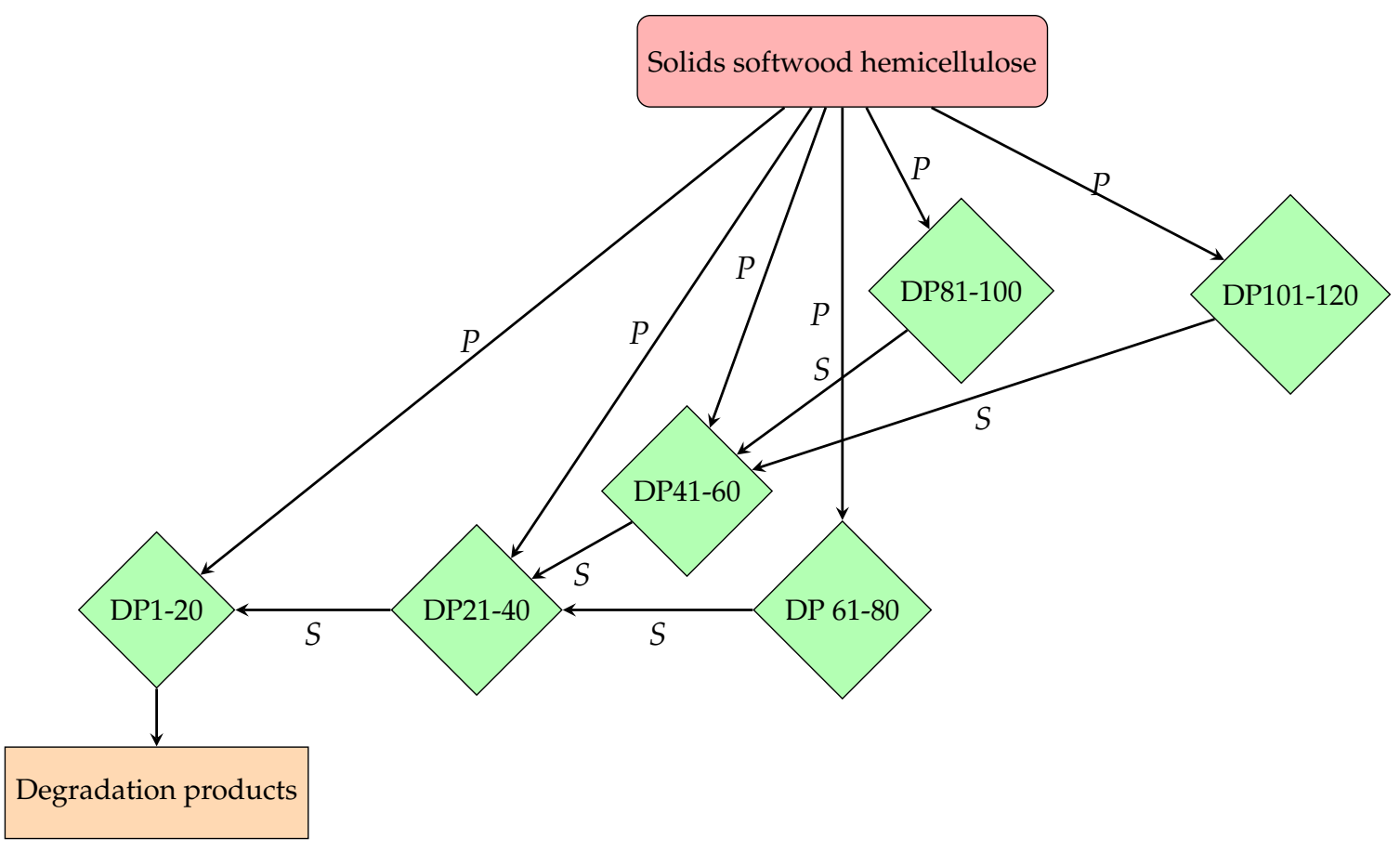

Figure S6: Mechanism of hemicellulose molar mass evolution in the range of 8 intervals including soluble oligomer with DP1-120, insoluble softwood hemicellulose, and degradation products. $P$ is a primary pathway to produce soluble hemicellulose from insoluble wood; $S$ is a secondary pathway to produce smaller soluble oligomers from a primary oligomer. 
Table S3: Molar mass and SEC retention time by DP.

\begin{tabular}{|c|c|c|c|}
\hline $\begin{array}{l}\text { Interval } \\
\text { number }\end{array}$ & $\begin{array}{l}\text { Degree of poly- } \\
\text { merization }\end{array}$ & $\begin{array}{l}\text { Molar mass } \\
(\mathrm{g} / \mathrm{mol})\end{array}$ & $\begin{array}{l}\text { Retention } \\
\text { time (min) }\end{array}$ \\
\hline 1 & N.A. & 126.1 & 61.4 \\
\hline \multirow[t]{3}{*}{2} & 1 & 173.1 & 60.1 \\
\hline & 10 & $1,568.9$ & 51.6 \\
\hline & 20 & $3,119.8$ & 48.9 \\
\hline \multirow[t]{2}{*}{3} & 30 & $4,670.7$ & 47.3 \\
\hline & 40 & $6,221.6$ & 46.2 \\
\hline \multirow[t]{2}{*}{4} & 50 & $7,772.5$ & 45.3 \\
\hline & 60 & $9,323.4$ & 44.6 \\
\hline \multirow[t]{2}{*}{5} & 70 & $10,874.3$ & 44.0 \\
\hline & 80 & $12,425.2$ & 43.5 \\
\hline \multirow[t]{2}{*}{6} & 90 & $13,976.1$ & 43.1 \\
\hline & 100 & $15,527.0$ & 42.7 \\
\hline \multirow[t]{2}{*}{7} & 110 & $17,077.9$ & 42.3 \\
\hline & 120 & $18,628.8$ & 41.9 \\
\hline
\end{tabular}



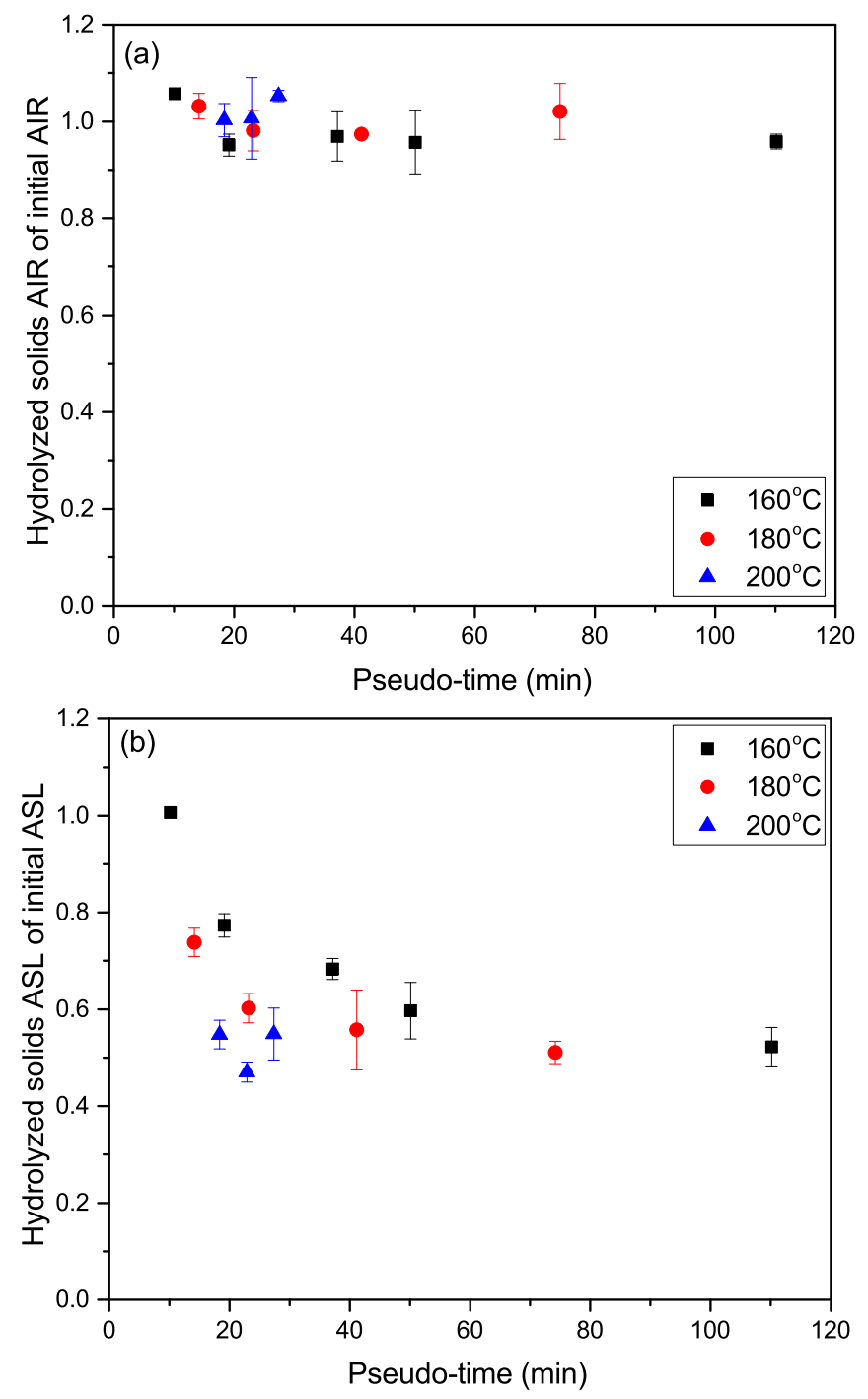

Figure S7: Hydrolyzed solids (a) Acid insoluble residue (AIR) relative to initial AIR and (b) Acid soluble lignin (ASL) relative to initial ASL in $25 \mathrm{~g}$ of o.d. softwood chip fines at $160{ }^{\circ} \mathrm{C}, 180{ }^{\circ} \mathrm{C}$ and $200^{\circ} \mathrm{C}$ as a function of pseudo-time. Error bars are the standard deviation of 2-3 replicates. 
Table S4: Composition of softwood on o.d. biomass basis and average hemicellulose monomer molar mass. The standard deviation is calculated from 4-6 replicates of each batch.

\begin{tabular}{|c|c|c|c|c|c|c|c|c|c|c|c|c|c|}
\hline $\begin{array}{l}\text { Batches } \\
\text { of soft- } \\
\text { wood }\end{array}$ & $\begin{array}{l}\text { Extractive } \\
(\%)\end{array}$ & $\begin{array}{l}\text { Sugar } \\
(\%)\end{array}$ & $\begin{array}{l}\text { Arabinan } \\
(\%)\end{array}$ & $\begin{array}{l}\text { Galactan } \\
(\%)\end{array}$ & $\begin{array}{l}\text { Total } \\
\text { glucan } \\
(\%)\end{array}$ & $\begin{array}{l}\text { Estimated } \\
\text { hemicellu- } \\
\text { lose glucan } \\
(\%)\end{array}$ & $\begin{array}{l}\text { Xylan } \\
(\%)\end{array}$ & $\begin{array}{l}\text { Mannan } \\
(\%)\end{array}$ & $\begin{array}{l}\text { Acid } \\
\text { insoluble } \\
\text { residue } \\
(\%)\end{array}$ & $\begin{array}{l}\text { Acid } \\
\text { soluble } \\
\text { lignin } \\
(\%)\end{array}$ & $\begin{array}{l}\text { Total } \\
\text { mass } \\
\text { balance } \\
(\%)\end{array}$ & $\begin{array}{l}\text { Hemicellulos } \\
\text { monomer } \\
\text { molar } \\
\text { mass (g/- } \\
\text { mol) }\end{array}$ & $\begin{array}{l}\text { e Average } \\
\text { hemicel- } \\
\text { lulose } \\
\text { monomer } \\
\text { molar mass } \\
(\mathrm{g} / \mathrm{mol})\end{array}$ \\
\hline Average & 3.92 & 63.03 & 1.49 & 3.43 & 42.30 & 9.84 & 5.96 & 9.84 & 27.45 & 4.35 & 98.74 & 172.84 & 173.09 \\
\hline $\begin{array}{l}\text { Standard } \\
\text { deviation }\end{array}$ & 0.53 & 3.28 & 0.13 & 0.17 & 3.13 & & 0.18 & 0.87 & 0.42 & 1.15 & 2.32 & & \\
\hline Average & 3.33 & 65.76 & 1.45 & 2.77 & 45.13 & 10.75 & 5.67 & 10.75 & 26.67 & 5.24 & 101.01 & 173.34 & \\
\hline $\begin{array}{l}\text { Standard } \\
\text { deviation }\end{array}$ & 0.19 & 1.30 & 0.05 & 0.09 & 1.07 & & 0.36 & 0.54 & 1.25 & 1.95 & 1.70 & & \\
\hline
\end{tabular}


To account for the difference between free monosaccharides and those present in oligosaccharides, due to condensation of water (Equation S1), an anhydro correction factor was applied in Equation S2. This method is adapted from Greenwood et al. (2015).

$$
\mathrm{G}_{n}+\mathrm{H}^{+}+\mathrm{H}_{2} \mathrm{O} \rightarrow 2 \mathrm{G}_{\frac{n}{2}}+\mathrm{H}^{+}
$$

where $G_{n}$ is the hemicellulose with $\mathrm{DP}=n$ and $G_{\frac{n}{2}}$ is the hemicellulose with $\mathrm{DP}=\frac{n}{2}$.

$$
C_{S, n}=C_{n, \sec } \times \frac{M_{w, n}-M_{w a t e r}}{M_{w, n}}
$$

where $C_{n, \text { sec }}$ is the hemicellulose concentration of $\mathrm{DP}=n$ calculated from SEC in $\mathrm{g} / \mathrm{L}$; $M_{w, n}$ is the molar mass of polymer with $\mathrm{DP}=n \mathrm{in} \mathrm{g} / \mathrm{mol} ; M_{w a t e r}$ is the molar mass of water in $\mathrm{g} / \mathrm{mol}$.

A similar correction must be applied for degradation products in interval 1. It was assumed that hydroxymethylfurfural $\left(\mathrm{HMF}, M_{w, h m f}=126.11 \mathrm{~g} / \mathrm{mol}\right.$ ) was the main component. The dehydration process removes 3 molecules of water (Equation S3). The mass correction factor is given by Equation S4.

$$
\begin{gathered}
\mathrm{HO}\left(\mathrm{C}_{6} \mathrm{H}_{10} \mathrm{O}_{5}\right) \mathrm{H} \rightarrow \mathrm{C}_{6} \mathrm{H}_{6} \mathrm{O}_{3}+3 \mathrm{H}_{2} \mathrm{O} \\
C_{h m f}=C_{h m f, s e c} \times \frac{M_{w, h m f}+3 \times M_{\text {water }}}{M_{w, h m f}}
\end{gathered}
$$


where $C_{h m f, s e c}$ is the concentration of HMF calculated from SEC in $\mathrm{g} / \mathrm{L} ; M_{w, h m f}$ is the molar mass of HMF in $\mathrm{g} / \mathrm{mol} ; M_{\text {water }}$ is the molar mass of water in $\mathrm{g} / \mathrm{mol}$.

The matrix form of governing equations for the population balance model is listed.

$$
\left(\begin{array}{l}
\frac{\mathrm{d} C_{1}}{\mathrm{~d} t_{p}} \\
\frac{\mathrm{d} C_{2}}{\mathrm{~d} t_{p}} \\
\frac{\mathrm{d} C_{3}}{\mathrm{~d} t_{p}} \\
\frac{\mathrm{d} C_{4}}{\mathrm{~d} t_{p}} \\
\frac{\mathrm{d} C_{5}}{\mathrm{~d} t_{p}} \\
\frac{\mathrm{d} C_{6}}{\mathrm{~d} t_{p}} \\
\frac{\mathrm{d} C_{7}}{\mathrm{~d} t_{p}} \\
\frac{\mathrm{d} C_{8}}{\mathrm{~d} t_{p}}
\end{array}\right)=\left(\begin{array}{cccccccc}
0 & k_{4} & 0 & 0 & 0 & 0 & 0 & 0 \\
0 & -k_{4} & k_{1}(2)^{k_{2}} & 0 & 0 & 0 & 0 & \frac{1}{6} k_{3} \\
0 & 0 & -k_{1}(2)^{k_{2}} & k_{1}(3)^{k_{2}} & k_{1}(4)^{k_{2}} & 0 & 0 & \frac{1}{6} k_{3} \\
0 & 0 & 0 & -k_{1}(3)^{k_{2}} & 0 & k_{1}(5)^{k_{2}} & k_{1}(6)^{k_{2}} & \frac{1}{6} k_{3} \\
0 & 0 & 0 & 0 & -k_{1}(4)^{k_{2}} & 0 & 0 & \frac{1}{6} k_{3} \\
0 & 0 & 0 & 0 & 0 & -k_{1}(5)^{k_{2}} & 0 & \frac{1}{6} k_{3} \\
0 & 0 & 0 & 0 & 0 & 0 & -k_{1}(6)^{k_{2}} & \frac{1}{6} k_{3} \\
0 & 0 & 0 & 0 & 0 & 0 & 0 & -k_{3}
\end{array}\right)\left(\begin{array}{l}
C_{1} \\
C_{2} \\
C_{3} \\
C_{4} \\
C_{5} \\
C_{6} \\
C_{7} \\
C_{8}
\end{array}\right)
$$




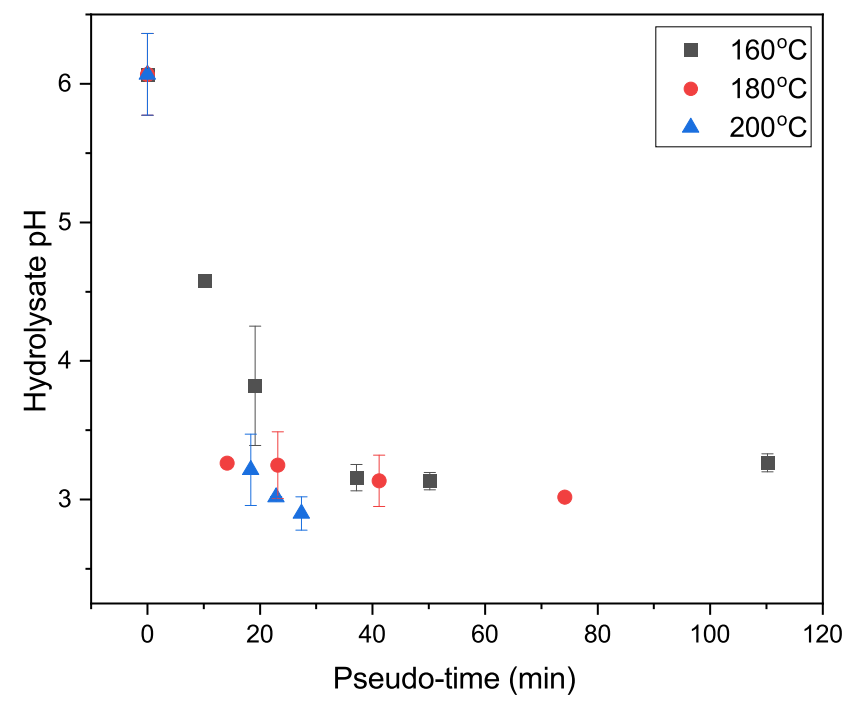

Figure S8: Hydrolysate $\mathrm{pH}$ as a function of pseudo-time (min) and temperature. Error bars are the standard deviation of 2-3 replicates. 

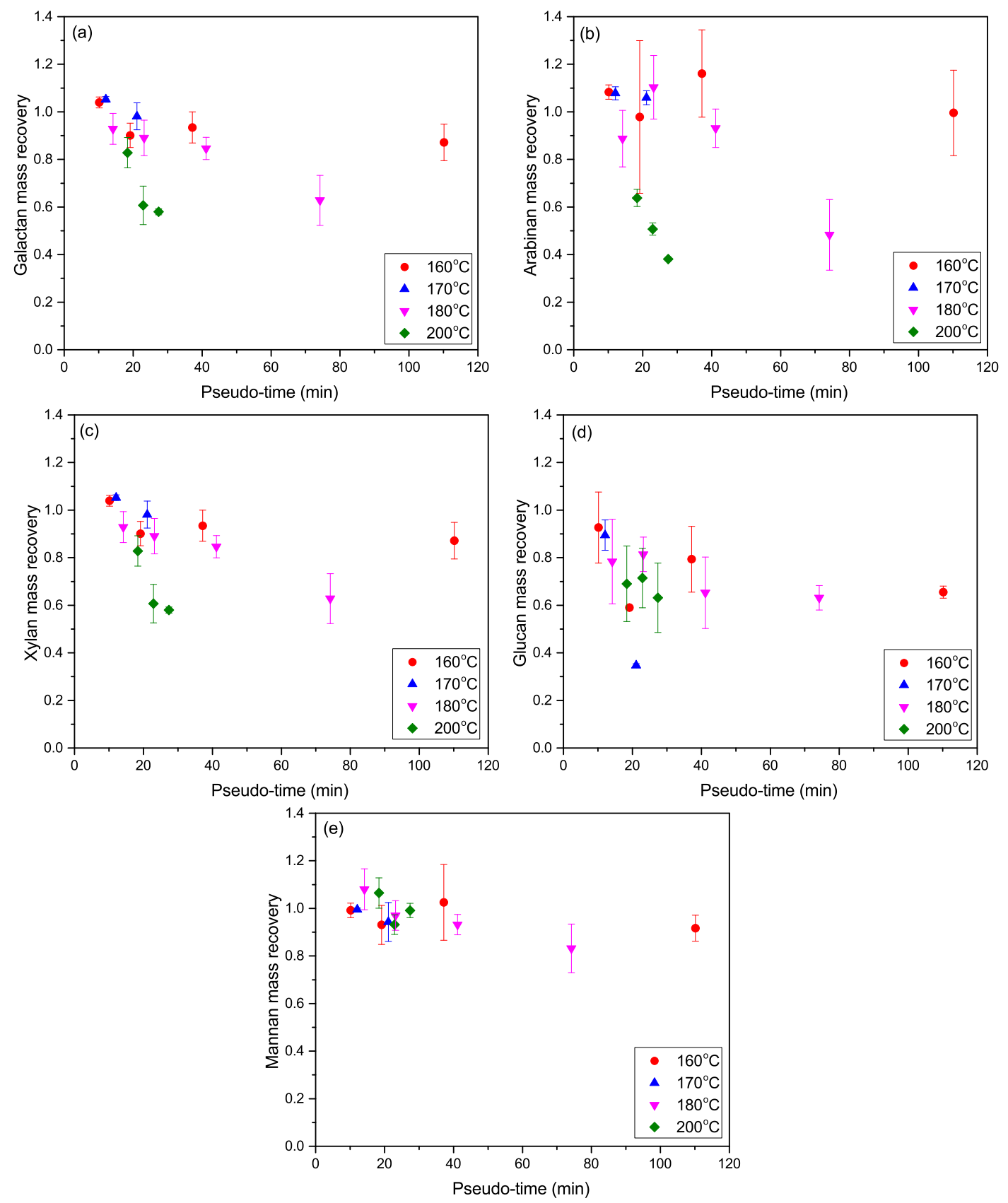

Figure S9: Hemicellulose mass recovery (summation of hydrolysate hemicellulose and prehydrolyzed solids hemicellulose) from hydrolysate and pretreated solids at $160^{\circ} \mathrm{C}$ to $200^{\circ} \mathrm{C}$ (a) Galactan (b) Arabinan (c) Xylan (d) Glucan (e) Mannan. Error bars are the standard deviation of 2-3 replicates. 

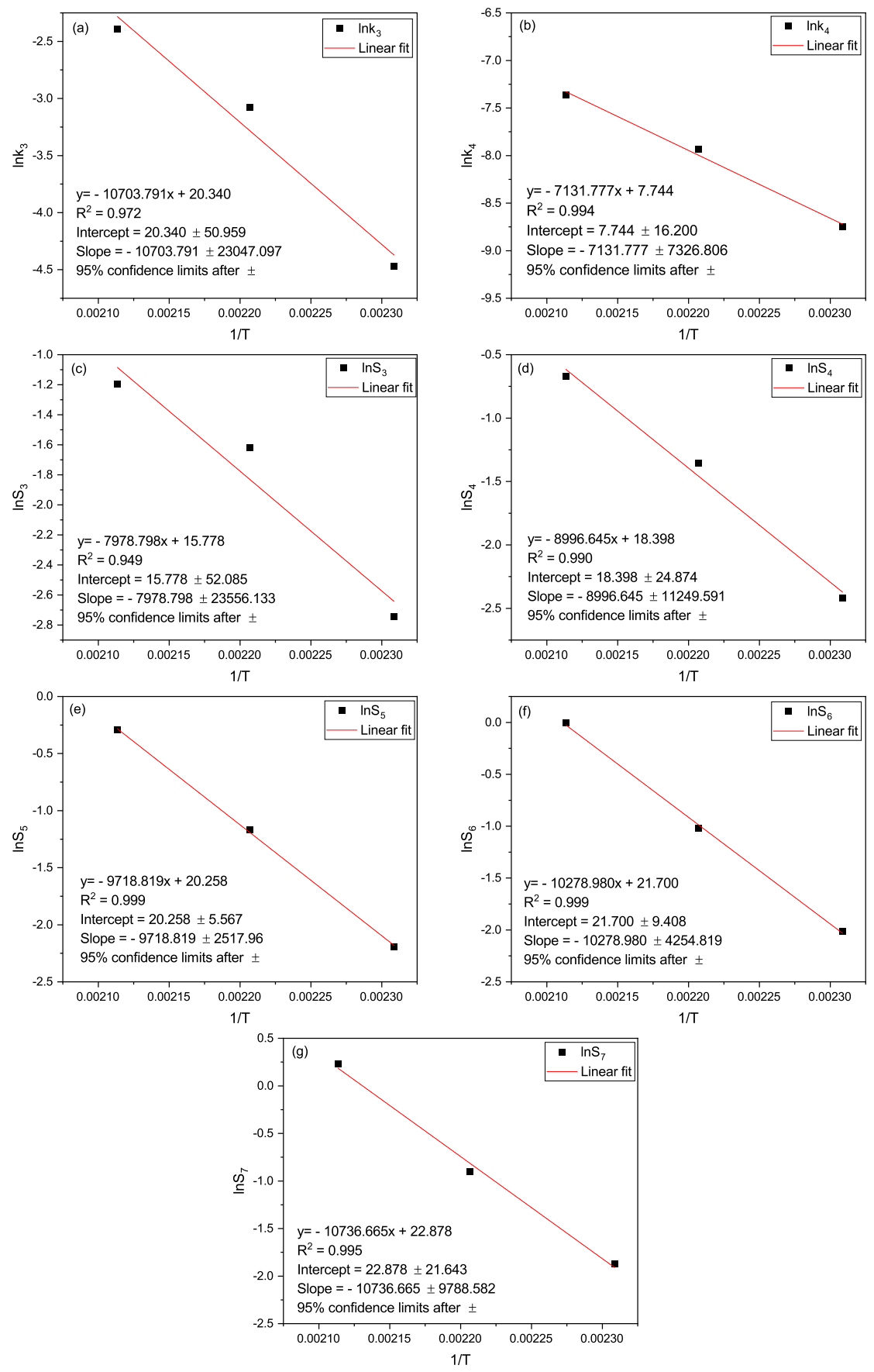

Figure S10: Linear regression for activation energy determination of (a) interval 8, (b) interval 1 and 2, (c) interval 3, (d) interval 4, (e) interval 5, (f) interval 6, and (g) interval 7. 
Table S5: Comparison of hemicellulose/wood hydrolysis activation energies reported in literature.

\begin{tabular}{|c|c|c|c|c|c|c|c|}
\hline Year & Author & Substrate & $\begin{array}{l}\text { Hydrolysis con- } \\
\text { dition }\end{array}$ & $\begin{array}{l}\text { Tempera- } \\
\text { ture }\left({ }^{\circ} \mathrm{C}\right)\end{array}$ & $\begin{array}{l}\text { Bond } \\
\text { breaking } \\
\text { mode }\end{array}$ & $\mathrm{Ea}\left(\mathrm{kJ} \cdot \mathrm{mol}^{-1}\right)$ & $\mathrm{A}\left(\mathrm{L} \cdot \mathrm{mol}^{-1} \cdot \mathrm{s}^{-1}\right)$ \\
\hline 2008 & Xu et al. (2008) & $\begin{array}{l}\text { O-acetyl } \\
\text { galactogluco- } \\
\text { mannans }\end{array}$ & $\begin{array}{l}1 \mathrm{M} \text { hydrochloric } \\
\text { acid } \mathrm{pH} \text { of } 1 \text { to } 2\end{array}$ & $25-90$ & Random & 150.3 & $6.09 \times 10^{21}$ \\
\hline 2012 & Visuri et al. (2012) & $\begin{array}{l}\text { Galactogluco- } \\
\text { mannan }\end{array}$ & $\begin{array}{l}\text { Hot water } \mathrm{pH} \text { of } \\
3.8 \text { to } 4.2\end{array}$ & $\begin{array}{l}150,160 \\
170\end{array}$ & Random & $102 \pm 20$ & $3.74 \times 10^{11}$ \\
\hline 2015 & Greenwood et al. (2015) & $\begin{array}{l}\text { Sugarcane } \\
\text { bagasse }\end{array}$ & $\begin{array}{l}0.5 \% \quad \text { sulfuric } \\
\text { acid }\end{array}$ & $110-170$ & Random & $\begin{array}{l}\text { solid: 191.29; soluble: } \\
\text { 93.42; degradation:154.68 }\end{array}$ & N.A. \\
\hline 2016 & Ahmad et al. (2016) & Birch wood & Hot water & 210 & Random & 134.4 & $3.7 \times 10^{15}$ \\
\hline 2020 & $\begin{array}{l}\text { This } \\
\text { work }\end{array}$ & Softwood & Hot water & $\begin{array}{l}160,180, \\
200\end{array}$ & $\begin{array}{l}\text { Random } \\
\text { / Middle }\end{array}$ & $\begin{array}{lrr} & \text { solid: } 89.00 ; & \text { soluble: } \\
66.34 & -89.27 ; & \text { degrada- } \\
\text { tion: } 59.30\end{array}$ & $\begin{array}{l}\text { solid: } \quad 1.14 \times 10^{7} ; \quad \text { solu- } \\
\text { ble: } 1.19 \times 10^{5}-1.44 \times 10^{8} \text {; } \\
\text { degradation: } 38.47\end{array}$ \\
\hline
\end{tabular}




\section{References}

Ahmad, Waqar, Kuitunen, Susanna, Sixta, Herbert, \& Alopaeus, Ville. 2015. Population balance based modeling of changes in cellulose molecular weight distribution during ageing. Cellulose, 22(1), 151-163.

Ahmad, Waqar, Kuitunen, Susanna, Borrega, Marc, \& Alopaeus, Ville. 2016. Physicochemical Modeling for Hot Water Extraction of Birch Wood. Industrial \& Engineering Chemistry Research, 55(42), 11062-11073.

Basedow, Arno Max, Ebert, Klaus Heinrich, \& Ederer, Hanns Josef. 1978. Kinetic studies on the acid hydrolysis of dextran. Macromolecules, 11(4), 774-781.

Greenwood, Ava A, Farrell, Troy W, Zhang, Zhanying, \& O'Hara, Ian M. 2015. A novel population balance model for the dilute acid hydrolysis of hemicellulose. Biotechnology for biofuels, 8(1), 26-39.

Visuri, Juha A, Song, Tao, Kuitunen, Susanna, \& Alopaeus, Ville. 2012. Model for degradation of galactoglucomannan in hot water extraction conditions. Industrial \& Engineering Chemistry Research, 51(31), 10338-10344.

Xu, Chunlin, Pranovich, Andrey, Vähäsalo, Lari, Hemming, Jarl, Holmbom, Bjarne, Schols, Henk A, \& Willför, Stefan. 2008. Kinetics of acid hydrolysis of water-soluble spruce O-acetyl galactoglucomannans. Journal of agricultural and food chemistry, 56(7), 2429-2435. 